AperTO - Archivio Istituzionale Open Access dell'Università di Torino

\title{
Bergmann-Bianchi identities in field theories
}

\section{This is the author's manuscript}

Original Citation:

Availability:

This version is available http://hdl.handle.net/2318/68609

since 2017-11-27T09:39:29Z

Publisher:

AIP Publishing

Terms of use:

Open Access

Anyone can freely access the full text of works made available as "Open Access". Works made available under a Creative Commons license can be used according to the terms and conditions of said license. Use of all other works requires consent of the right holder (author or publisher) if not exempted from copyright protection by the applicable law. 


\title{
Bergman-Bianchi identities in field theories*
}

\author{
M. Francaviglia, M. Palese and E. Winterroth \\ Department of Mathematics, University of Torino \\ via C. Alberto 10, I-10123 Torino, Italy \\ E-MAIL: MARCELLA.PALESE@UNITO.IT
}

\begin{abstract}
We relate the generalized Bergman-Bianchi identities for Lagrangian field theories on gauge-natural bundles with the kernel of the associated gauge-natural Jacobi morphism.
\end{abstract}

2000 MSC: 58A20,58A32,58E30,58E40,58J10,58J70.

Key words: gauge-natural bundles, Bergman-Bianchi identities, Jacobi morphisms.

\section{The Bergman-Bianchi morphism}

Our general framework is the calculus of variations on finite order gaugenatural bundles $[3,8]$. Such geometric structures have been widely recognized to suitably describe so-called gauge-natural field theories, i.e. physical theories in which right-invariant infinitesimal automorphisms of the structure bundle $\boldsymbol{P}$ uniquely define the transformation laws of the fields themselves (see e.g. [4] and references quoted therein). We shall in particular consider finite order variational sequences on gauge-natural bundles, whereby foundamental objects of calculus of variations such as Lagrangians, Euler-Lagrange and Jacobi morphisms are conveniently represented as quotient morphisms (see e.g. $[9,6])$. For basic notions and fixing notation we refer to $[1,3,4,5,6,8,11]$ and references therein.

\footnotetext{
*Work partially supported by MIUR (PRIN 2003) and University of Torino
} 
Recall that generalized Bergman-Bianchi identities for field theories are necessary and (locally) sufficient conditions for the Noether conserved current to be not only closed but also the divergence of a skew-symmetric (tensor) density along solutions of the Euler-Lagrange equations [1]. It was also stressed that in the general theory of relativity these identities coincide with the contracted Bianchi identities for the curvature tensor of the pseudo-Riemannian metric.

Let $\boldsymbol{Y}_{\zeta}$ be a gauge-natural bundle and let $\lambda$ be a gauge-natural Lagrangian $[4,8]$ on the $s$-th order prolongation $J_{s} \boldsymbol{Y}_{\zeta}$. Let $\mathcal{A}^{(r, k)}$ be the vector bundle of right-invariant principal automorphisms of the underlying principal structure bundle $\boldsymbol{P}$. In the following we shall consider variation vector fields which are vertical parts of gauge-natural lifts of a given $\bar{\Xi} \in \mathcal{A}^{(r, k)}$. Let $\mathcal{C}_{2 s}^{*}\left[\mathcal{A}^{(r, k)}\right] \simeq$ $J_{2 s+1} \mathcal{A}^{(r, k)} \times{ }_{J_{2 s} \mathcal{A}^{(r, k)}} V J_{2 s} \mathcal{A}^{(r, k)}$. By a slight abuse of notation, we denote by $\mathfrak{G}(\bar{\Xi})_{V}$ the vertical part - with respect to the contact structure induced by the projections $J_{s+1} \boldsymbol{Y}_{\zeta} \rightarrow J_{s} \boldsymbol{Y}_{\zeta}$ - of (jet prolongation of) the gauge-natural lift $\mathfrak{G}(\bar{\Xi})[3,4,5]$. We set

$$
\left.\omega\left(\lambda, \mathfrak{G}(\bar{\Xi})_{V}\right) \equiv £_{\Xi}\right\rfloor \mathcal{E}_{n}(\lambda): J_{2 s} \boldsymbol{Y}_{\zeta} \rightarrow \mathcal{C}_{2 s}^{*}\left[\mathcal{A}^{(r, k)}\right] \otimes \mathcal{C}_{0}^{*}\left[\mathcal{A}^{(r, k)}\right] \wedge\left(\wedge{ }^{n} T^{*} \boldsymbol{X}\right),
$$

where $£ \equiv$ is the Lie derivative operator acting on sections of the gaugenatural bundle [5], 」 is the interior product and $\mathcal{E}_{n}(\lambda)$ is the generalized Euler-Lagrange morphism associated with $\lambda[6]$. The morphism $\omega\left(\lambda, \mathfrak{G}(\bar{\Xi})_{V}\right)$ so defined is a generalized Lagrangian associated with the field equations of the original Lagrangian $\lambda$ and it has been considered in applications e.g. in General Relativity. By the linearity of $£$ we can regard $\omega\left(\lambda, \mathfrak{G}(\bar{\Xi})_{V}\right)$ as the extended morphism defined on $J_{2 s} \boldsymbol{Y}_{\zeta} \underset{X}{\times} J_{2 s} \mathcal{A}^{(r, k)}$. We have $D_{H} \omega\left(\lambda, \mathfrak{G}(\bar{\Xi})_{V}\right)=$ 0 , where $D_{H}$ is the exterior differential; thus, as a consequence of a global decomposition formula for vertical morphisms [7], we can state the following [11].

Lemma 1 Let $\omega\left(\lambda, \mathfrak{G}(\bar{\Xi})_{V}\right)$ be as above. On the domain of $\omega\left(\lambda, \mathfrak{G}(\bar{\Xi})_{V}\right)$ we have (up to pull-backs):

$$
\omega\left(\lambda, \mathfrak{G}(\bar{\Xi})_{V}\right)=\beta\left(\lambda, \mathfrak{G}(\bar{\Xi})_{V}\right)+F_{\omega\left(\lambda, \mathfrak{G}(\bar{\Xi})_{V}\right)},
$$

where

$$
\beta\left(\lambda, \mathfrak{G}(\bar{\Xi})_{V}\right) \equiv E_{\omega\left(\lambda, \mathfrak{G}(\bar{\Xi})_{V}\right)}
$$

and, locally, $F_{\omega\left(\lambda, \mathfrak{G}(\overline{\bar{\Xi}})_{V}\right)}=D_{H} M_{\omega\left(\lambda, \mathfrak{G}(\overline{\bar{\Xi}})_{V}\right)}$. 
Definition 1 We call the global morphism $\beta\left(\lambda, \mathfrak{G}(\bar{\Xi})_{V}\right):=E_{\omega\left(\lambda, \mathfrak{G}(\bar{\Xi})_{V}\right)}$ the generalized Bergman-Bianchi morphism associated with the Lagrangian $\lambda$ and the variation vector field $\bar{\Xi}$.

Let $\mathfrak{K}$ be the kernel of $\mathcal{J}\left(\lambda, \mathfrak{G}(\bar{\Xi})_{V}\right)$. We have the following characterization of the Bergman-Bianchi identities for gauge-natural theories [11].

Theorem 1 The generalized Bergman-Bianchi morphism is globally vanishing for the variation vector field $\bar{\Xi}$ if and only if $\delta_{\mathfrak{G}}^{2} \lambda \equiv \mathcal{J}\left(\lambda, \mathfrak{G}(\bar{\Xi})_{V}\right)=0$, i.e. if and only if $\mathfrak{G}(\bar{\Xi})_{V} \in \mathfrak{K}$.

From now on we shall write $\omega(\lambda, \mathfrak{K})$ to denote $\omega\left(\lambda, \mathfrak{G}(\overline{\bar{\Xi}})_{V}\right)$ when $\mathfrak{G}(\bar{\Xi})_{V}$ belongs to $\mathfrak{K}$. Analogously for $\beta$ and other morphisms.

First of all let us make the following important consideration. Let $\mathcal{L}_{j_{s}}$ छ be the variational Lie derivative operator [6] acting on gerneralized variational morphisms.

Proposition 1 For each $\bar{\Xi} \in \mathcal{A}^{(r, k)}$ such that $\bar{\Xi}_{V} \in \mathfrak{K}$, we have

$$
\left.\mathcal{L}_{j_{s} \bar{\Xi}_{H}} \omega(\lambda, \mathfrak{K})=-D_{H}\left(-j_{s} \Xi_{\Xi}\right\rfloor p_{D_{V} \omega(\lambda, \mathfrak{K})}\right) .
$$

Proof. We have

$$
\mathcal{L}_{j_{s} \bar{\Xi}_{V}} \omega(\lambda, \mathfrak{K})=\mathcal{L}_{j_{s} \bar{\Xi}_{V}} \mathcal{L}_{j_{s} \bar{\Xi}} \lambda=\mathcal{L}_{j_{s}\left[\bar{\Xi}_{V}, \bar{\Xi}_{H}\right]} \lambda .
$$

On the other hand it is also easy to verify that

$$
\mathcal{L}_{j_{s} \bar{\Xi}_{H}} \omega(\lambda, \mathfrak{K})=\mathcal{L}_{j_{s}\left[\bar{\Xi}_{H}, \bar{\Xi}_{V}\right]} \lambda=-\mathcal{L}_{j_{s} \bar{\Xi}_{V}} \omega(\lambda, \mathfrak{K}) .
$$

Since

$$
\begin{aligned}
& \left.\left.\mathcal{L}_{j_{s} \Xi_{V}} \omega(\lambda, \mathfrak{K})=-£_{\Xi}\right\rfloor \mathcal{E}_{n}(\omega(\lambda, \mathfrak{K}))+D_{H}\left(-j_{s} £_{\Xi}\right\rfloor p_{D_{V} \omega(\lambda, \mathfrak{K})}\right)= \\
& \left.=\beta(\lambda, \mathfrak{K})+D_{H}\left(-j_{s} £ \equiv\right\rfloor p_{D_{V} \omega(\lambda, \mathfrak{K})}\right),
\end{aligned}
$$

from the Theorem above we get the assertion.

The new generalized Lagrangian $\omega(\lambda, \mathfrak{K})$ is gauge-natural invariant too, i.e. $\mathcal{L}_{j_{s} \Xi} \omega(\lambda, \mathfrak{K})=0$.

Even more, we can state the following

Proposition 2 Let $\bar{\Xi}_{V} \in \mathfrak{K}$. We have

$$
\mathcal{L}_{j_{s} \bar{\Xi}_{H}} \omega(\lambda, \mathfrak{K})=0 .
$$


Corollary 1 Let $\bar{\Xi}_{V} \in \mathfrak{K}$. We have the covariant conservation law

$$
\left.D_{H}\left(-j_{s} £_{\Xi}\right\rfloor p_{D_{V} \omega(\lambda, \Re)}\right)=0 .
$$

Definition 2 We define the covariantly conserved current

$$
\left.\mathcal{H}(\lambda, \mathfrak{K})=-j_{s} £_{\Xi}\right\rfloor p_{D_{V} \omega(\lambda, \mathfrak{K})},
$$

to be a Hamiltonian form for $\omega(\lambda, \mathfrak{K})$ (in the sense of $[10]$ ).

\section{References}

[1] P.G. Bergman: Conservation Laws in General relativity as the Generators of Coordinate Transformations, Phys. Rev. 112 (1) (1958) 287-289.

[2] B. Casciaro, M. Francaviglia: A new variational characterization of Jacobi fields along geodesics. Ann. Mat. Pura Appl. (4) 172 (1997), 219-228.

[3] D.J. EcK: Gauge-natural bundles and generalized gauge theories, Mem. Amer. Math. Soc. 247 (1981) 1-48.

[4] L. Fatibene, M. Francaviglia: Natural and gauge natural formalism for classical field theories. A geometric perspective including spinors and gauge theories; Kluwer Academic Publishers, Dordrecht, 2003.

[5] L. Fatibene, M. Francaviglia, M. Palese: Conservation laws and variational sequences in gauge-natural theories, Math. Proc. Camb. Phil. Soc. 130 (2001) 555569.

[6] M. Francaviglia, M. Palese, R. Vitolo: Symmetries in Finite Order Variational Sequences, Czech. Math. J. 52(127) (2002) 197-213.

[7] I. KolÁř: A Geometrical Version of the Higher Order Hamilton Formalism in Fibred Manifolds, J. Geom. Phys., 1 (2) (1984) 127-137.

[8] I. Kolář, P.W. Michor, J. Slovák: Natural Operations in Differential Geometry, (Springer-Verlag, N.Y., 1993).

[9] D. Krupka: Variational Sequences on Finite Order Jet Spaces, Proc. Diff. Geom. and its Appl. (Brno, 1989); J. Janyška, D. Krupka eds.; World Scientific (Singapore, 1990) 236-254.

[10] L. Mangiarotti, G. Sardanashvily: Connections in Classical and Quantum Field Theory, (World Scientific, Singapore, 2000).

[11] M. Palese, E. Winterroth: Global Generalized Bianchi Identities for Invariant Variational Problems on Gauge-natural Bundles, to appear in Arch. Math. (Brno). 